\title{
Autologous CLL1-CD33 Compound CAR T Cells
}

National Cancer Institute

\section{Source}

National Cancer Institute. Autologous CLL1-CD33 Compound CAR T Cells. NCI

Thesaurus. Code C157281.

Autologous T-lymphocytes transduced with a lentiviral vector expressing a compound chimeric antigen receptor (CCAR) containing two CARs, one specific for the CD33 antigen and one specific for the C-type-lectin-like molecule-1 (CLL1; C-type lectin domain family 12 member A; CLEC12A), with potential immunomodulating and antineoplastic activities. Upon administration, the CD33/CLL1-specific CARs lentiviral vector-transduced autologous T-lymphocytes, expressing both the anti-CD33 CAR and the anti-CLL1 CAR on their surfaces, specifically and simultaneously targ et and bind to CD33- and CLL1expressing tumor cells, with their anti-CD33 CAR and their anti-CLL1 CAR, respectively. This induces selective toxicity in tumor cells that express the CD33 antigen and the CLL1 antigen. CD33 is expressed on normal non-pluripotent hematopoietic stem cells and is overexpressed on myeloid leukemia cells. CLL1, a member of the C-type lectin/C-type lectin-like domain (CT L/CT LD) superfamily, is overexpressed in leukemic stem cells (LSCS) and plays an important role in disease progression and relapse for myeloid malignancies. 